Proc. XIX International School of Semiconducting Compounds, Jaszowiec 1990

\title{
THE DYNAMIC DIELECTRIC FUNCTION "ANOMALY" FOR HgSe:Ga AND HgSe:Fe*
}

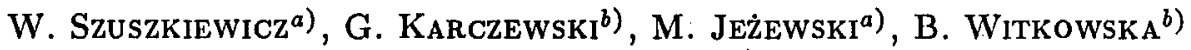 \\ AND A. MYCIELSKI ${ }^{b}$ ) \\ a) Institute of Experimental Physics, Warszawa University, Hoża 69, 00-681 Warszawa, \\ Poland

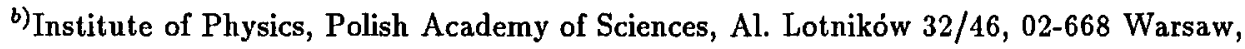 \\ Poland
}

(Received August 8, 1990)

\begin{abstract}
Reflectivity spectra of $\mathrm{HgSe}$ crystals highly doped with $\mathrm{Ga}$ and Fe, respectively, were investigated in the spectral region close to the absorption edge at temperatures between $12 \mathrm{~K}$ and $295 \mathrm{~K}$. From the analysis of the structure observed in reflectivity the temperature dependence of the Fermi energy was determined for both kinds of crystals.
\end{abstract}

PACS numbers: 75.50.Pp, 78.20.Ci, 78.30.Fs

HgSe is a well known zero-gap semiconductor (see e.g., [1]). The optical properties of a semiconductor may be described by the complex Dynamic Dielectric Function (DDF), dependent only on the light frequency. One of the important contributions to the DDF in the case of an $n$-type zero-gap semiconductor is the term related to the heavy hole valence band - conduction band optical transitions [2]. Due to this term the real part of the DDF has a peak in the vicinity of the Fermi energy $E_{\mathrm{F}}$. This "anomaly" of the DDF produces peaks in the energy dependence of both refraction index and the reflectivity in the spectral region close to $E_{\mathrm{F}}$. Until now the reflectivity spectra for $\mathrm{HgSe}$ have investigated for a relatively low electron concentration range $[3,4]$.

The goal of this paper was to analyse the infrared reflectivity for HgSe highly doped with different impurities. Two different kinds of crystals containing Ga and Fe, respectively, were used for optical measurements. As it is known, the Fe impurity in $\mathrm{HgSe}$ acts as a resonant donor located at about $E_{\mathrm{Fe}}=200 \mathrm{meV}$ above the bottom of the conduction band (e.g. $[5,6]$ and the references therein). The electron concentration for these samples was equal to about $N=5 \times 10^{18} \mathrm{~cm}^{-3}$

*This work was supported in part by CPBP 01.06 . 


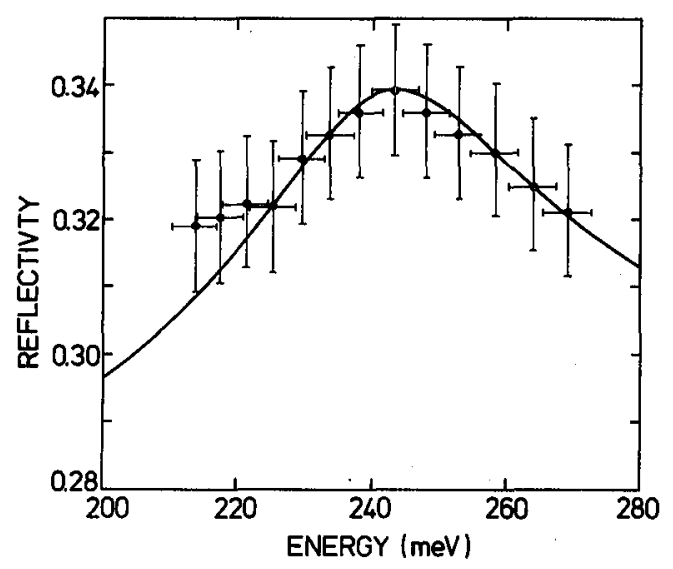

Fig.1.: Reflectivity versus energy for $\mathrm{HgSe}$ doped with $\mathrm{Ga}$ at $\mathrm{T}=72 \mathrm{~K}$. The electron concentration for this sample determined from the Hall measurements at $\mathrm{T}=77 \mathrm{~K}$ is $N=4.69 \times 10^{18} \mathrm{~cm}^{-3}$. The solid line corresponds to the theoretical reflectivity spectrum. The Fermi energy $E_{\mathrm{F}}=223 \mathrm{meV}$ gives the best fit of this spectrum to the shown experimental points.

at low temperatures. Samples after mechanical polishing and chemical etching in a $5 \%$ bromine methanol solution were mounted on the cold finger of a helium cryostat. The lowest temperature available was equal to $12 \mathrm{~K}$.

The reflectivity spectra at different temperatures were measured in the energy range close to the Fermi energy, (spectral region near $5-6 \mu \mathrm{m}$ ), where the DDF "anomaly" should exist. The spectral resolution was of the order of about $2 \%$ of the incident light energy. The experimental reflectivity values were always below the values predicted by the theory. Under the circumstances (we were interested in the reflectivity peak position only) the measured reflectivity spectra were normalised to the theoretical ones. For a given sample the Fermi energy value $E_{\mathrm{F}}$ was used as a fitting parameter at every temperature. An exemple of such a fit is shown in Fig. 1. The quality of the fits at the lowest temperatures was not as good as that shown in this figure. The reason was that the observed structures were not as sharp as those predicted by the theory, in particular for the Fe-doped samples. Figure 2 shows the $E_{F}$ temperature dependence, optically determined by the method presented in Fig. 1, for two of our samples. As expected, the energy position of the DDF "anomaly" in $\mathrm{HgSe}: \mathrm{Ga}$ moves as a result of the temperature dependence of $E_{\mathrm{F}}$ and can be satisfactorily described by the theory. The $E_{\mathrm{F}}$ value for HgSe:Fe on the contrary is in practice temperature independent at temperatures below $80 \mathrm{~K}$. This is due to the Fermi energy pinning to the $\mathrm{Fe}^{2+}$ level at low temperatures.

At higher temperatures $E_{\mathrm{F}}$ increases with temperature, as has been demonstrated e.g. on the basis of transnmission measurements $[7,8]$. Preliminary results taken on the Fe-doped samples with relatively smaller iron concentration $\left(N_{\mathrm{Fe}}\right.$ 


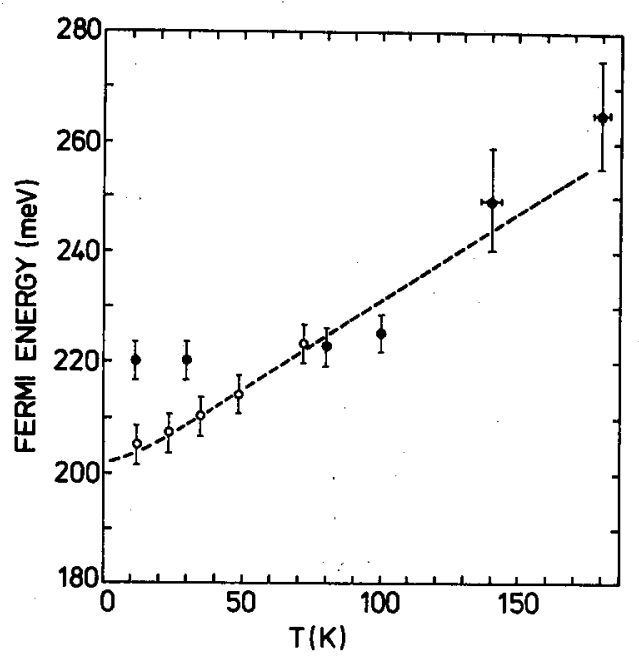

Fig.2. Temperature dependence of the Fermi energy $E_{F}$ determined from the fits similar to that shown in Fig. 1 for two of our samples. o - experimental data for HgSe:Ga crystal mentioned in the text, - - experimental data for $\mathrm{HgSe}: \mathrm{Fe}$ corresponding to the iron concentration equal to $N_{\mathrm{Fe}}=1.7 \times 10^{20} \mathrm{~cm}^{-3}$. The electron concentration for both samples is expected to be very similar and ranging from $4.5 \times 10^{18} \mathrm{~cm}^{-3}$ to $5 \times 10^{18} \mathrm{~cm}^{-3}$ at low temperatures. The broken line corresponds to the theoretical temperature dependence of the Fermi energy for the Ga-doped crystal.

ranging from $5 \times 10^{1} 8 \mathrm{~cm}^{-3}$ to $4 \times 10^{1} 9 \mathrm{~cm}^{-3}$ ) correspond to the Fermi energy equal to about $E_{\mathrm{F}}=195 \mathrm{meV}$ at low temperatures. This value also remains constant at least below $77 \mathrm{~K}$ within experimental error. Concluding, we would like to stress that the optically determined value of the Fermi energy for all $\mathrm{HgSe}: \mathrm{Fe}$ samples does not depend on the temperature in the low temperature range, in contrast to the $\mathrm{HgSe}: \mathrm{Ga}$ case. A slight increase of this value with increasing $\mathrm{Fe}$ concentration has been observed.

\section{References}

[1] M. Dobrowolska, W. Dobrowolski, A. Mycielski, Solid State Commun. 34, 441 (1980).

[2] W. Szuszkiewicz, A.M. Witowski, M. Grynberg, Phys. Status Solidi B 87, 637 (1978).

[3] A. Manabe, H. Noguchi, A, Mitsuishi, Phys. Status Solidi B 90, 157 (1980).

[4] A.M. Witowski, M. Grynberg, Phys. Status Solidi B 100, 389 (1980). 
[5] A. Mycielski, B. Kowalski, B.A. Orłowski, M. Dobrowolska, M. Arciszewska, W. Dobrowolski, J.M. Baranowski, J. Phys. C. 19, 3605 (1986).

[6] F.S. Pool, J. Kossut, U. Dębska, R. Reifenberger, Phys. Rev. B 35, 3900 (1987).

[7] W. Szuszkiewicz, Qian Dingrong, M. Jeżewski, Zhang Jiaming, A. Mycielski, Acta Phys. Pol. A77, 159 (1990).

[8] W. Szuszkiewicz, Qian Dingrong, C. Julien, Zhang Jiaming, M. Jeżewski, M. Balkanski, A. Mycielski, J. Cryst. Growth 101, 507 (1990). 